\title{
Syndemics of syphilis, HCV infection, and methamphetamine use along the east coast of China
}

Meizhen Liao' ${ }^{1}$, Dianmin Kang ${ }^{1 *}$, Xiaorun Tao ${ }^{1}$, Catherine Cox², Yuesheng Qian' ${ }^{1}$ Guoyong Wang ${ }^{1}$, Cui Yang ${ }^{3}$, XiaoYan Zhu', Na Zhang ${ }^{1}$, Zhenqiang $\mathrm{Bi}^{1}$ and Yujiang $\mathrm{Jia}^{4^{*}}$

\begin{abstract}
Background: An upsurge in club drug use has been observed in recent years in some cities of China, especially methamphetamine, which is quickly replacing heroin to become the most widespread drug across the nation. This study investigated the type of drugs used, syphilis and hepatitis C virus (HCV) infection and the correlates for syphilis, $\mathrm{HCV}$ and unprotected commercial sex behavior among drug users in two cities along the east coast of China.

Methods: A cross-sectional survey conducted in 2010 provided demographics, sexual and drug use behaviors, HIV knowledge and the utilization of intervention services among drug users. Blood samples were tested for HIV, syphilis, and HCV infection.

Results: Of 805 eligible participants, $0.2 \%$ were infected with HIV, 3.7\% with HCV, and $9.6 \%$ with syphilis. Of the participants, $96.6 \%$ were methamphetamine users, $11.9 \%$ reported ever having used $\geq 2$ types of these drugs, and $11.4 \%$ reported ever injecting drugs. In the multivariable logistic regression analysis, participants infected with syphilis were more likely to be female (adjusted odds ratio $(A O R)=2.8,95 \%$ confidence interval $(C \mathrm{C}): 1.2-6.5)$, have ever had commercial sex in the past 12 months ( $A O R=2.0,95 \% \mathrm{Cl}: 1.0-3.9$ ), be infected with HCV (AOR=12.1, 95\% Cl: 4.1-20.3) and less likely to have ever had sex with regular partners in the past 12 months ( $\mathrm{AOR}=0.2,95 \% \mathrm{Cl}: 0.1-0.6$ ). Participants infected with HCV were more likely to have ever injected drugs ( $\mathrm{AOR}=2.7,95 \% \mathrm{Cl}: 1.1-6.5$ ) and be infected with syphilis (AOR=8.0,95\% Cl: 3.5-18.0). Participants who had unprotected sex with commercial sex partners in the last sexual encounter were more likely to be female ( $A O R=2.9,95 \%$ Cl:1.7-4.9), have middle school or lower level education ( $\mathrm{AOR}=3.4,95 \% \mathrm{Cl}: 2.0-5.5$ ), never have received intervention in the last year ( $\mathrm{AOR}=2.1,95 \%$ Cl:1.2-3.6) and be infected with syphilis (AOR=4.2, 95\% Cl:2.4-7.4).

Conclusions: Methamphetamine is the predominant drug used among the drug users, the prevalence of syphilis and HCV infection are alarmingly high, and unprotected commercial sex was common among this group. The findings highlight the need for effective, multifaceted interventions addressing sexual and drug use-related risky behaviors among this group. Further research is needed to better understand the causal pathway of the syndemics.
\end{abstract}

Keywords: Syphilis, HCV, Methamphetamine, Sexual behavior, Drug user

\footnotetext{
*Correspondence: dmkang@sohu.com; jiayj@aol.com

${ }^{1}$ Institution for AIDS/STD Control and Prevention \& Shandong Key Laboratory

for Epidemic Disease Control and Prevention, Shandong Center for Disease

Control and Prevention, Jinan, Shandong Province 250014, P R China

${ }^{4}$ Department of Preventive Medicine, Vanderbilt University, Nashville, TN

37232, USA

Full list of author information is available at the end of the article
} 


\section{Background}

Historically, injection drug use, mainly heroin, was concentrated in the rural areas of southwestern and northwestern China, and has been the major contributor to the HIV/ AIDS epidemic in this country [1-3]. However, in recent years, China has seen an upsurge in club drug use, especially methamphetamine, which is quickly replacing heroin to become the most widespread illicit drug across the nation [4-7]. Shandong is one of the provinces confronting the rapidly emerging club drug use. One of the cities along the east coast has been named the "ice city", for its common use of club drugs, especially among young people. MA and other club drugs are often used in entertainment venue settings, such as hotels, Bars, KTV and tea houses. Club drugs were perceived as a pastime, an entertainment additive without any stigma attached to it, rather than the highly stigmatized traditional substance abuse. Methamphetamine can be smoked, snorted, injected or orally ingested. These drugs meet the psychological characteristics of young people, e.g., curiosity, wonder, and excitement $[8,9]$. Synthetic drug use fuels the transmission of HIV/other STDs (sexually transmitted diseases) [10,11]. Empirical studies from other countries have demonstrated an association between synthetic drug use and risky sexual behaviors that include unprotected anal and vaginal intercourse, and sexual activities with multiple partners, as well as an association between club drug use and HIV/other STDs [12-15]. Among the 780,000 estimated HIV/AIDS cases in 2011,63.9\% were infected through sexual transmission, and $17.4 \%$ through male-to-male contact, which has emerged as one of the major transmission routes in China [16]. New types of drugs, termed synthetic drugs or club drug users, could play a significant role in fueling the epidemics of HIV and other STDs without an effective action taken in a timely fashion [7,17].

China faces the emerging syndemics of HIV, other STDs, and synthetic drugs $[18,19]$. Studies have been conducted focusing on HIV and other STDs, however, the research on club drugs and its impact on HIV and other STDs remains scarce. Both the extent of the epidemic of club drug use and its social consequences are not yet fully recognized [17]. Limited information is available on the syndemics of HIV/other STDs, Hepatitis C Virus (HCV), and club drugs in China [20]. This study investigated the type of drugs, syphilis and hepatitis $\mathrm{C}$ virus $(\mathrm{HCV})$ infection and the correlates for syphilis, HCV and unprotected commercial sex behavior among drug users in Jining City and Qingdao City, Shandong Province of China.

\section{Methods}

\section{Recruitments and participants}

A cross-sectional survey was conducted in Jining and Qingdao, Shandong Province in 2010. Prior to the recruitment of the participants of the survey, we conducted informative research including in-depth interviews with key informants to gather the background information among drug users, e.g., the venues to access them, selection of the candidates of the first group for interview, and sampling frame. All potential participants were invited for an eligibility assessment. Recruitment criteria required that participants be willing to complete the study, and self-report having used drugs in the last year. Participants were approached from drug user gathering venues such as bars, night clubs, bathhouses, saunas, public parks, outdoor cruising areas, and HIV testing sites. After these initial participants were approached and interviewed after an eligibility assessment, we asked the participants to refer their peers to attend the study. A mixed recruitment method, including community outreach, venue-based recruitment, and peer referrals, was applied in the study. Structured questionnaire based interviews were conducted by trained health professionals in a carefully selected clinic in each city. Voluntary participation, anonymity and confidentiality were ensured for all participants. The trained interviewer also conducted some of the interviews within the community or venues such as hotels or bars because some of the participants were not willing to visit the clinics. The experienced study staff explained the study purpose and protocol including voluntary and anonymity of participation, confidentiality and security of data collection, and safety of blood sample collection to all potential participants. An informed consent was received from all eligible participants. The study was approved by the Institutional Review Board (IRB) of Shandong Center for Disease Control and Prevention.

\section{Measures}

Questionnaire-based interviews provided information including demographics (age, marital status, ethnicity, residency, education), sexual and drug use behaviors (sex behavior with regular and commercial partners, condom use with clients or regular sex partners, types of drug use and injection history), HIV knowledge, HIV testing, and HIV-related prevention services. Participants who reported using various types of drugs in the last year were defined as methamphetamine, heroin, cocaine, cannabis, morphine, pethidine, ketamine, MAMD (Ecstasy), and amphetamine users. The regular sex partner refers to their spouse if married or the sex partners that are not married but live together.

Level of HIV knowledge was assessed using eight questions regarding the modes of HIV transmission and prevention, as well as misperception. All questions were weighted equally. Responses to these questions were combined into an overall score for each of the correct and incorrect answers. These scores were then stratified into two groups, according to whether six or more appropriate responses were given to the eight 
questions. The use of HIV-related prevention services was assessed by whether participants had ever received one of the five indicators related to relevant prevention services in the past 12 months (peer education, HIV counseling and testing, community drug maintenance treatment, needle and syringe provision \& exchange, or received free condoms).

Blood samples were collected and tested for HIV, syphilis and $\mathrm{HCV}$. Two screening tests were used to diagnose HIV: an enzyme-linked immunosorbent assay and a confirmatory test using the HIV-1/2 Western Blot immune assay (HIV Blot 2.2 WB; Genelabs Diagnostics, Singapore). Syphilis was diagnosed using rapid plasma reagin $\left(R P R^{T M}\right)$ and a Passive Particle Agglutination Test for Detection of Antibodies to Treponema pallidum (TPPA $^{\mathrm{TM}}$, Rong Sheng Biostix Inc, Shanghai, China). Two screening tests using an enzyme-linked immunoassay (Beijing Wantai Biological Medicine Company, China and Xiamen Xinchuang Industrial Scientific, China) were performed to determine $\mathrm{HCV}$ status. All of the participants were informed of their testing results of HIV, syphilis and $\mathrm{HCV}$ by trained health professionals. All of the notification of testing results and referrals for positive individuals to subsequent prevention, counseling, and treatment services were also provided in a confidential manner using a prior signed unique code.

\section{Statistical analyses}

Questionnaire-based data and biological results were recorded into the EpiData software (EpiData 3.0 for Windows, the EpiDate Association Odense, Denmark). All analyses were performed with the SPSS ${ }^{\circ}$ software (Version 15.0; SPSS Inc., Chicago, Illinois, USA). The prevalence rates of syphilis, HCV and unprotected sex were calculated by demographic characteristics, drug use and sexual behaviors, and utilization of HIV-related prevention services. Univariate analyses were performed for the independent variables of demographic, behavioral, and biological variables. A multivariable logistic regression model was constructed. Both adjusted odds ratios (AOR) and $95 \%$ confidence intervals (CIs) were calculated for each explanatory variable in the final models. All potential co-linearity, potential confounding, and effect modification were examined and taken into consideration in the final models. Statistical significance for the variables was determined with $\mathrm{p}<0.05$ in the model.

\section{Results}

\section{Characteristics of participants}

Of 805 eligible participants, $82.8 \%$ were male; $28.0 \%$ single, $70.2 \%$ currently married or cohabitating; the median age was 30 years with a range from 16 to 67 years; $86.5 \%$ were local residents; $64.2 \%$ received middle school or a lower level of education; only $3.6 \%$ belonged to non-Han ethnic groups; $66.8 \%$ correctly answered at least 6 out of 8 questions on HIV transmission, prevention and misperceptions; $16.1 \%$ received HIV-related intervention in the past 12 months; $4.7 \%$ had a test for HIV in the past 12 months while $52.6 \%$ knew their HIV test result (Table 1).

\section{Drug use, sexual behaviors and correlates for unprotected sex}

Of the participants, $96.6 \%(\mathrm{n}=778)$ were methamphetamine users, $6.3 \%(n=51)$ ketamine users, $4.5 \%(n=36)$ MAMD users, $4.0 \%(\mathrm{n}=32)$ amphetamine users and $3.2 \%(\mathrm{n}=26)$ heroin users. Of the participants, $11.9 \%(\mathrm{n}=96)$ reported having ever used $\geq 2$ types of these drugs and $11.4 \%$ $(n=92)$ reported having ever injected drugs. Of the participants, $98.8 \%$ reported ever having sex with regular sex partners, with $74.7 \%$ never having used condoms in the past 12 months and $92.7 \%$ reporting no condom use in the last sexual encounter. $52.5 \%$ of participants reported having commercial sex, with $89.9 \%$ reporting inconsistent condom use in the past 12 months and $75.2 \%$ reporting no condom use in the last sexual encounter (Table 1). Participants who have ever had unprotected sex with commercial sex partners in the last sexual encounter were more likely to be female, have received middle school or a lower level of education, have never received an intervention in the last year and be infected with syphilis (Table 2).

\section{Correlates for syphilis, HCV infection}

Of the participants, $0.2 \%(\mathrm{n}=2)$ were infected with HIV, 9.6\% $(\mathrm{n}=77)$ with syphilis and $3.7 \%(\mathrm{n}=30)$ with HCV. $1.4 \%$ $(\mathrm{n}=11)$ were infected with both syphilis and HCV. All syphilis cases $(n=77)$ were methamphetamine users with 2 of them also ketamine and amphetamine users (Table 1). The participants infected with syphilis were more likely to be female, have ever had commercial sex in the past 12 months, be infected with HCV and less likely to have ever had sex with regular sex partners in the past 12 months. Participants infected with HCV were more likely to have ever injected drugs and be co-infected with syphilis (Table 2).

\section{Discussion}

This is the first to report on the assessment of the syndemics of HIV, syphilis, HCV, and MA use among drug users in China. This study found alarmingly high rates of syphilis (9.6\%), HCV infection (3.7\%), and MA use (96.6\%) among drug users in the two cities along the east coast of China with historically low HIV prevalence. Syphilis and $\mathrm{HCV}$ infection predicted each other, and being female was a common correlate for syphilis and unprotected sex. The findings of this study contribute to the understanding of the emergent role of club drug abuse on the HIV/syphilis epidemic and its association with risky sexual behavior in the two cities of Shandong Province. 
Table 1 Demographic characteristics, drug use and sexual behaviors, and utilization of HIV-related prevention services among club drug users in Shandong Province, China

\begin{tabular}{|c|c|c|c|c|c|c|c|c|}
\hline \multirow[t]{2}{*}{ Factors } & \multicolumn{2}{|c|}{ Participants } & \multicolumn{2}{|c|}{ Syphilis } & \multicolumn{2}{|c|}{$\mathrm{HCV}$} & \multicolumn{2}{|c|}{ Unprotected sex* } \\
\hline & $\mathrm{N}=805$ & $\%$ & $\mathrm{n}=77$ & $\%$ & $n=30$ & $\%$ & $\mathrm{~N}=211$ & $\%$ \\
\hline Age (years; $\bar{x} \pm S D$ ) & \multicolumn{2}{|c|}{$30 \pm 9.1$} & \multicolumn{2}{|c|}{$32.1 \pm 11.4$} & \multicolumn{2}{|c|}{$29.8 \pm 10.1$} & \multicolumn{2}{|c|}{$29.9 \pm 9.2$} \\
\hline$\geq 18$ & 285 & 35.4 & 27 & 9.5 & 11 & 3.9 & 72 & 49.0 \\
\hline $25-35$ & 281 & 34.9 & 20 & 7.1 & 11 & 3.9 & 62 & 44.9 \\
\hline $35-45$ & 183 & 22.7 & 21 & 11.5 & 6 & 3.3 & 57 & 54.3 \\
\hline$\geq 45$ & 56 & 7.0 & 9 & 16.1 & 2 & 3.6 & 20 & 60.6 \\
\hline \multicolumn{9}{|l|}{ Gender } \\
\hline Male & 662 & 82.2 & 52 & 7.9 & 26 & 3.9 & 176 & 37.6 \\
\hline Female & 143 & 17.8 & 25 & 17.5 & 4 & 2.8 & 35 & 53.3 \\
\hline \multicolumn{9}{|l|}{ Marital status } \\
\hline Single & 225 & 28.0 & 21 & 9.3 & 9 & 4.0 & 67 & 47.2 \\
\hline Married or cohabiting & 565 & 70.2 & 52 & 9.2 & 20 & 3.5 & 139 & 51.5 \\
\hline Divorced or widowed & 15 & 1.8 & 4 & 26.7 & 1 & 6.7 & 5 & 45.5 \\
\hline \multicolumn{9}{|l|}{ Education } \\
\hline Middle school or lower & 517 & 64.2 & 49 & 9.5 & 15 & 2.9 & 179 & 58.5 \\
\hline High school or higher & 288 & 35.8 & 28 & 9.7 & 15 & 5.2 & 32 & 27.4 \\
\hline \multicolumn{9}{|l|}{ Types of drugs ${ }^{\#}$} \\
\hline Heroin & 26 & 3.2 & 0 & 0.0 & 3 & 11.5 & 2 & 28.6 \\
\hline Cocaine & 1 & 0.1 & 0 & 0.0 & 0 & 0.0 & 1 & 100 \\
\hline Opium & 0 & 0 & 0 & 0.0 & 0 & 0.0 & 0 & 0 \\
\hline Cannabis & 4 & 0.5 & 0 & 0.0 & 0 & 0.0 & 1 & 33.3 \\
\hline Morphine & 1 & 0.1 & 0 & 0.0 & 0 & 0.0 & 0 & 0 \\
\hline Methamphetamine & 778 & 96.6 & 77 & 9.9 & 28 & 3.6 & 207 & 49.9 \\
\hline Pethidine & 0 & 0 & 0 & 0.0 & 0 & 0.0 & 0 & 0 \\
\hline Ketamine & 51 & 6.3 & 1 & 2.0 & 2 & 3.9 & 2 & 28.6 \\
\hline MAMD & 36 & 4.5 & 0 & 0.0 & 0 & 0.0 & 1 & 20.0 \\
\hline Amphetamine & 32 & 4.0 & 1 & 3.1 & 3 & 9.4 & 2 & 40.0 \\
\hline \multicolumn{9}{|l|}{ Multi-drug use (types) } \\
\hline$<2$ & 709 & 88.1 & 75 & 10.6 & 25 & 3.5 & 208 & 51.0 \\
\hline$\geq 2$ & 96 & 11.9 & 2 & 2.1 & 5 & 5.2 & 3 & 20.0 \\
\hline \multicolumn{9}{|l|}{ Had ever injected } \\
\hline Yes & 92 & 11.4 & 8 & 8.7 & 9 & 9.8 & 11 & 44.0 \\
\hline No & 713 & 88.6 & 69 & 9.7 & 21 & 2.9 & 200 & 50.3 \\
\hline \multicolumn{9}{|c|}{ Had ever sex with regular sex partners in last year } \\
\hline Yes & 558 & 98.8 & 49 & 8.8 & 20 & 3.6 & 138 & 51.9 \\
\hline No & 7 & 1.2 & 3 & 42.9 & 0 & 0.0 & 1 & 25.0 \\
\hline \multicolumn{9}{|c|}{ Condom use with regular sex partners in last year } \\
\hline Always & 21 & 3.8 & 0 & 0.0 & 1 & 4.8 & 0 & 0 \\
\hline Sometimes & 120 & 21.5 & 6 & 5.0 & 2 & 1.7 & 4 & 11.4 \\
\hline Never & 417 & 74.7 & 43 & 10.3 & 17 & 4.1 & 134 & 58.8 \\
\hline \multicolumn{9}{|c|}{ Condom use with regular sex partners in last sex } \\
\hline Yes & 41 & 7.3 & 1 & 2.4 & 1 & 2.4 & 0 & 0 \\
\hline No & 517 & 92.7 & 49 & 8.8 & 19 & 3.7 & 138 & 54.5 \\
\hline
\end{tabular}


Table 1 Demographic characteristics, drug use and sexual behaviors, and utilization of HIV-related prevention services among club drug users in Shandong Province, China (Continued)

\begin{tabular}{|c|c|c|c|c|c|c|c|c|}
\hline \multicolumn{9}{|c|}{ Had ever sex with commercial sex partners in last year } \\
\hline Yes & 423 & 52.5 & 49 & 11.6 & 13 & 3.1 & - & - \\
\hline No & 382 & 47.5 & 28 & 7.3 & 17 & 4.5 & - & - \\
\hline \multicolumn{9}{|c|}{ Condom use with commercial sex partners in last year } \\
\hline Always & 43 & 10.1 & 2 & 4.7 & 1 & 2.3 & - & - \\
\hline Sometimes & 169 & 40.0 & 23 & 13.6 & 3 & 1.8 & - & - \\
\hline Never & 211 & 49.9 & 24 & 11.4 & 3 & 1.4 & - & - \\
\hline \multicolumn{9}{|c|}{ Condom use with commercial sex partners in last sex } \\
\hline Yes & 105 & 24.8 & 6 & 5.7 & 3 & 2.9 & - & - \\
\hline No & 318 & 75.2 & 43 & 13.5 & 4 & 1.3 & - & - \\
\hline \multicolumn{9}{|c|}{ Ever received intervention } \\
\hline Yes & 130 & 16.1 & 14 & 10.8 & 5 & 3.8 & 29 & 34.1 \\
\hline No & 675 & 83.9 & 63 & 9.3 & 25 & 3.7 & 182 & 53.8 \\
\hline \multicolumn{9}{|l|}{ Syphilis/HCV infection } \\
\hline Syphilis Positive & 77 & 9.6 & - & - & 11 & 14.3 & 24 & 56.2 \\
\hline HCV Positive & 30 & 3.7 & 11 & 36.7 & - & - & 3 & 42.9 \\
\hline Syphilis/HCV Negative & 709 & 88.1 & - & - & - & - & 186 & 26.2 \\
\hline \multicolumn{9}{|l|}{ HIV knowledge score } \\
\hline Score $<6$ & 267 & 33.2 & 31 & 11.6 & 7 & 2.6 & 155 & 72.1 \\
\hline Score $\geq 6$ & 538 & 66.8 & 46 & 8.6 & 23 & 4.3 & 56 & 26.9 \\
\hline
\end{tabular}

Subtotals do not add up to $100 \%$ for some variables because of missing values.

*Unprotected sex means no condom use with commercial sex partners in the last year.

"The factor of "types of drugs" was multi-choice variables, the percentages will not add up to $100 \%$.

This study showed that nearly $10 \%$ of participants were infected with syphilis, and all of them were MA users. The syphilis prevalence among drug users was significantly higher than that of female sex workers (3.07\%), men who have sex with men $(4.69 \%)$, pregnant women $(0.33 \%)$ and unpaid blood donors $(0.08 \%)$ in the same year. The logistic analysis revealed that syphilis was independently associated with ever having had unprotected sex with commercial sex partners in the past 12 months. Moreover, an alarmingly high prevalence $(52.5 \%)$ of drug users self-reported ever having had sex with commercial sex partners, with $89.9 \%$ of these participants self-reporting inconsistent condom use in this study. MA use enhances sexual performance, sensitivity, and pleasure, resulting in a complexity of sexual partners and network, thereby increasing the risk of trauma from prolonged intercourse and failure to use condoms due to altered judgment and inhibition [21,22]. It is even more worrisome that club drug users will take a potential role in the epidemiologic bridge of HIV/syphilis transmission to their regular sex partners [22]. This study also showed that club drug users had a low condom use rate with their regular sex partners. Trust in a sex partner or worrying suspicion ever having had commercial sex behavior by their regular sex partner resulted in low condom use with their regular sex partners. This indicates a great risk in transmission and/or acquisition of HIV/STDs within the club drug abuse and sex networks.

Results also showed participants who have ever had unprotected sex with commercial sex partners in the last sexual encounter were more likely to be female and the participants infected with syphilis were more likely to be female. Being female is the most vulnerable group for possible syphilis infection. In this study, of all participants, $17.8 \%(n=143)$ were female, among which $65.0 \%(n=93)$ reported having commercial sex. The syphilis prevalence was significantly higher among those females who have ever had commercial sex than those females who have never had commercial sex (22.5\% VS. 8.0\%). Our previous studies have shown the prevalence of syphilis infection increasing because of the club drug abuse among female sex workers (FSWs) in Qingdao [7]. However, the current intervention efforts are still focused on heroin, which played an important role in China's HIV/AIDS epidemic in the last decades, and the prevention infrastructure has not been strategized to tackle the newly emerging challenge of club drug abuse. In addition, this study also showed that only $4.7 \%$ of participants ever had a test for HIV while 52.6\% knew their HIV test result, and $16.1 \%$ had received intervention in the past 12 months. A low number of HIV tests and HIV-related prevention services reflected the less intervention efforts 
Table 2 Factors associated with syphilis and HCV infection among club drug users in Shandong, China

\begin{tabular}{|c|c|c|c|c|c|c|c|c|c|}
\hline \multirow[t]{2}{*}{ Factors } & \multicolumn{3}{|c|}{ Syphilis infection( $n=77$ ) } & \multicolumn{3}{|c|}{ HCV infection $(n=30)$} & \multicolumn{3}{|c|}{ Unprotected sex } \\
\hline & $\%$ & $\mathrm{OR}(95 \% \mathrm{Cl})$ & $\operatorname{AOR}(95 \% \mathrm{Cl})$ & $\%$ & OR(95\% Cl) & $\operatorname{AOR}(95 \% \mathrm{Cl})$ & $\%$ & OR(95\% Cl) & $\operatorname{AOR}(95 \% \mathrm{CI})$ \\
\hline \multicolumn{10}{|l|}{ Gender } \\
\hline Male & 7.9 & 1.0 & 1.0 & 3.9 & 1.0 & & 37.6 & 1.0 & 1.0 \\
\hline Female & 17.5 & $2.5(1.5-4.2)^{* *}$ & $2.8(1.2-6.5)^{*}$ & 2.8 & $0.7(0.2-2.0)$ & & 53.3 & $3.0(1.8-5.1)^{* *}$ & $2.9(1.7-4.9)^{* *}$ \\
\hline \multicolumn{10}{|l|}{ Education } \\
\hline Middle school or lower & 9.5 & 1.0 & & 2.9 & 1.0 & & 58.5 & $3.7(2.3-5.9)^{* *}$ & $3.4(2.0-5.5)^{* *}$ \\
\hline High school or higher & 9.7 & $1.0(0.6-1.7)$ & & 5.2 & $1.8(0.9-3.8)$ & & 27.4 & 1.0 & 1.0 \\
\hline \multicolumn{10}{|l|}{ Had ever injected } \\
\hline Yes & 8.7 & $0.9(0.4-1.9)$ & & 9.8 & $3.6(1.6-8.1)^{* *}$ & $2.7(1.1-6.5)^{*}$ & 44.0 & $0.8(0.4-1.7)$ & \\
\hline No & 9.7 & 1.0 & & 2.9 & 1.0 & 1.0 & 50.3 & 1.0 & \\
\hline \multicolumn{10}{|c|}{ Had ever sex with regular sex partners in last year } \\
\hline Yes & 8.8 & $0.1(0.1-0.6)^{* *}$ & $0.2(0.1-0.6)^{* *}$ & 3.6 & & & & & \\
\hline No & 42.9 & 1.0 & & 0.0 & & & & & \\
\hline \multicolumn{10}{|c|}{ Had ever sex with commercial sex partners in last year } \\
\hline Yes & 11.6 & $1.7(1.0-2.7)^{*}$ & $2.0(1.0-3.9)^{*}$ & 3.1 & $0.7(0.5-1.2)$ & & & & \\
\hline No & 7.3 & 1.0 & 1.0 & 4.5 & 1.0 & & & & \\
\hline \multicolumn{10}{|l|}{ Ever received intervention } \\
\hline Yes & 10.8 & 1.0 & & 3.8 & 1.0 & & 34.1 & 1.0 & 1.0 \\
\hline No & 9.3 & $0.9(0.5-1.6)$ & & 3.7 & $1(0.4-2.6)$ & & 53.8 & $2.3(1.4-3.7)^{* *}$ & $2.1(1.2-3.6)^{*}$ \\
\hline \multicolumn{10}{|l|}{ HCV infection } \\
\hline Yes & 36.7 & $6.2(2.8-13.6)^{* *}$ & $12.1(4.1-20.3)^{* *}$ & & & & 42.9 & $0.9(0.4-1.9)$ & \\
\hline No & 8.5 & 1.0 & 1.0 & & & & 45.4 & 1.0 & \\
\hline \multicolumn{10}{|l|}{ Syphilis } \\
\hline Yes & & & & 14.3 & $6.2(2.8-13.6)^{* *}$ & $8.0(3.5-18.0)^{* *}$ & 56.2 & $3.0(1.8-5.1)^{* *}$ & $4.2(2.4-7.4)^{* *}$ \\
\hline No & & & & 2.6 & 1.0 & & 27.9 & 1.0 & 1.0 \\
\hline
\end{tabular}

OR: Odds ratio; AOR: Adjusted odds ratio, controlled factors included study sites, age, sex, residency, ethnicity, education, marital status, sexual behavior, HIV-related prevention services; Cl: Confidence interval; NA: Not applicable; ${ }^{*} \mathrm{P}<0.05 ;{ }^{*} \mathrm{P}<0.01$.

targeted this group [1,23], and the low practice of VCT still remains a challenge among targeted drug users. Unprotected heterosexual contacts has been increasing proportionally in contribution of HIV transmission in China, therefore it is possible that the club drug abuse could further fuel this trend in the nation if no bettertargeted, effective intervention efforts are taken in a timely fashion [24].

Independent risk factors for $\mathrm{HCV}$ were injection drug users and co-infection with syphilis. According to this study, needle sharing of injection drug users could be a reasonable cause of the $\mathrm{HCV}$ infections. The findings underscore that harm reduction clear needle should be emphasized. Meanwhile, the high prevalence of syphilis demonstrated that a large number of drug users engaged in unprotected sex behaviors that may place them at high risk for HCV. HCV could be transmitted through parenteral exposures to contaminated blood, with efficiency through the use of injection drugs, e.g., sharing of needles or works [2,3]. Studies have revealed that $\mathrm{HCV}$ risk increases commensurate with increasing numbers of heterosexual sex partners [25]. Studies have found that common factors for $\mathrm{HCV}$ were associated with group sex, accompanied by STDs, the use of club drugs and other non-injection drugs during sex [26,27]. More research is needed to better understand the role of heterosexual transmission of HCV among this emerging group with a high risk for HIV and club drug use.

This study revealed that the prevalence of syphilis/HCV infection is alarmingly high and unprotected commercial sex was common among drug users. New types of drugs, especially MA, have become the predominant sources of drug abuse in these two cities in China. The findings of this study underscored the urgent need for comprehensive intervention strategies, including sex and drug-related interventions and HIV/syphilis/HCV control among this group. Methamphetamine is the predominant drug used among the drug users. We also should pay more attention to the intervention efforts to target syphilis prevention in FSWs, especially the drug using FSWs, and further research 
is needed to better understand the causal pathway of the syndemics, the driving forces for the high prevalence of syphilis, $\mathrm{HCV}$, and MA use.

We recognized the limitations of this study. Data collection relying on retrospective self-reports was subject to recall bias. The sensitive nature of the questions (related to sexual and drug use behaviors) may have led to information bias due to the social desirability of certain answers. We didn't collect participants' sexual preference in this study. The cross-sectional nature of the data also makes it impossible to establish any causality. It may be another limitation that the non-response or refusal of participation during the recruitment process could reduce the representativeness of the study participants. Non-response could be substantial in this study because drug use is illegal in China. Information on study participation was lacking. Moreover, the recruitment criterion to complete the study may lead to a selection bias. Therefore, the results of this study might not fully generalizable to drug users in other settings in China or different countries. Despite these limitations and possible biases, we feel the data highlights a prevention opportunity that cannot be ignored.

\section{Conclusions}

Synthetic drugs, especially methamphetamine, is the predominant drug used among the drug users in these two cities in China, the prevalence of syphilis and $\mathrm{HCV}$ infection are alarmingly high, and unprotected commercial sex was common among this group. The use of methamphetamine could fuel the epidemics of HIV, syphilis and other STDs in China. So far, no effective intervention programs are available targeting synthetic drug users in China. The findings highlight the need for effective, multifaceted interventions addressing sexual and drug use-related risky behaviors among this group. Further research is needed to better understand the causal pathway of the syndemics.

\section{Abbreviations \\ HCV: Hepatitis C virus; HIV: Human immunodeficiency virus; \\ MA: Methamphetamine; STD: Sexually transmitted disease; AIDS: Acquired immunodeficiency syndrome; Cl: Confidence interval; AOR: Adjusted odds ratio.}

\section{Competing interests}

The authors declare that they have no competing interests.

\section{Authors' contributions}

$M L, D K, X T, K Y, Y Q, G W, X Z, N Z$ and $Z B$ directed the study data collection and managed the data. ML, CC, CY, XZ, NZ and $Y J$ conducted the primary analyses and developed the first draft of the manuscript. YJ, ML, and DK provided interpretation to the analyses and wrote parts of the methods, results or discussion section or provided major editing. All authors contributed to the writing process before the final version was approved. All authors read and approved the final manuscript.

\section{Acknowledgements}

We are grateful to the study staff from the local Center for Disease Control and Prevention (CDC) for recruiting participants, and to participants for sharing their time and experiences. Special thanks to all subjects for their voluntary participation, and the owners of venues for their support in the preparation and implementation of the surveys.

\section{Author details}

${ }^{1}$ Institution for AIDS/STD Control and Prevention \& Shandong Key Laboratory for Epidemic Disease Control and Prevention, Shandong Center for Disease Control and Prevention, Jinan, Shandong Province 250014, P R China.

${ }^{2}$ Department of Epidemiology and Biostatistics, School of Public Health, University of Maryland College Park, College Park, MD 20742, USA.

${ }^{3}$ Department of Health, Behavior and Society, Johns Hopkins Bloomberg School of Public Health, Baltimore, MD 21205, USA. ${ }^{4}$ Department of Preventive Medicine, Vanderbilt University, Nashville, TN 37232, USA.

Received: 18 November 2012 Accepted: 7 February 2014 Published: 17 February 2014

\section{References}

1. The State Council AIDS Working Committee Office and the UN Theme Group on HIV/AIDS in China: A joint assessment of HIV/AIDS prevention, treatment and care in China. Beijing: China Ministry of Health; 2007.

2. Jia Y, Lu F, Zeng G, Sun X, Xiao Y, Lu L, Liu W, Ni M, Qu S, Li C, et al: Predictors of HIV infection and prevalence for syphilis infection among injection drug users in China: community-based surveys along major drug trafficking routes. Harm Reduction Journal 2008, 5:29.

3. Jia Y, Sun J, Fan L, Song D, Tian S, Yang Y, Jia M, Lu L, Sun X, Zhang S, et al: Estimates of HIV prevalence in a highly endemic area of China: Dehong Prefecture, Yunnan Province. Int J Epidemiol 2008, 37(6):1287-1296.

4. Farrell M, Marsden J, Ali R, Ling W: Methamphetamine: drug use and psychoses becomes a major public health issue in the Asia Pacific region. Addiction 2002, 97(7):771-772.

5. United Nations Office on Drugs and Crime (UNODC): 2008 World Drug Report. Vienna: United Nations Office on Drugs and Crime; 2009.

6. Kang D, Liao M, Jiang Z, Zhang X, Mao W, Zhang N, Tao X, Huang T, Bi Z, Aliyu $M$, et al: Commercial sex venues, syphilis and methamphetamine use among female sex workers. AIDS Care 2011, 23(Suppl 1):26-36.

7. Liao M, Jiang Z, Zhang X, Kang D, Bi Z, Liu X, Fu J, Zhang N, Mao W, Jiang $B$, et al: Syphilis and methamphetamine use among female sex workers in Shandong Province, China. Sex Transm Dis 2011, 38(1):57-62.

8. Parsons JT, Kelly BC, Weiser JD: Initiation into methamphetamine use for young gay and bisexual men. Drug Alcohol Depend 2007, 90(2-3):135-144.

9. Schilder AJ, Lampinen TM, Miller ML, Hogg RS: Crystal methamphetamine and ecstasy differ in relation to unsafe sex among young gay men. Can J Public Health 2005, 96(5):340-343.

10. China Drug Illicit Surveillance Center: Drug illicit surveillance report from 2005 to 2008. Beijing: China Drug Illicit Surveillance Center; 2009.

11. Colfax G, Guzman R: Club drugs and HIV infection: a review. Clin Infect Dis 2006, 42(10):1463-1469.

12. Jerome RC, Halkitis PN, Siconolfi DE: Club drug use, sexual behavior, and HIV seroconversion: a qualitative study of motivations. Subst Use Misuse 2009, 44(3):431-447.

13. Ministry of Health and Joint United Nations Programme on HIV/AIDS: 2011 Estimates for the HIV/AIDS Epidemic in China. Beijing: China Ministry of Health; 2012.

14. Yang $X$, Xia G: Causes and consequences of increasing club drug use in China: a descriptive assessment. Subst Use Misuse 2010, 45(1-2):224-239.

15. Potula R, Persidsky Y: Adding fuel to the fire: methamphetamine enhances HIV infection. Am J Pathol 2008, 172(6):1467-1470.

16. Liang $H$, Wang $X$, Chen $H$, Song L, Ye L, Wang SH, Wang YJ, Zhou L, Ho WZ: Methamphetamine enhances HIV infection of macrophages. Am J Pathol 2008, 172(6):1617-1624.

17. National Institute on Drug Abuse: Research Report Series. In Methamphetamine Abuse and Addiction. Edited by NIDA. Beijing: NIDA; 2006.

18. Boddiger D: Metamphetamine use linked to rising HIV transmission. Lancet 2005, 365(9466):1217-1218.

19. Wang N, Wang L, Wu Z, Guo W, Sun X, Poundstone K, Wang Y: Estimating the number of people living with HIV/AIDS in China: 2003-09. Int J Epidemiol 2010, 39(Suppl 2):ii21-ii28.

20. China CDC: National behavioral and biological Isurveillance report 2011. Beijing: National Center for AIDS/STD Control and Prevention, China CDC; 2012. 
21. Garofalo R, Mustanski BS, McKirnan DJ, Herrick A, Donenberg GR: Methamphetamine and young men who have sex with men: understanding patterns and correlates of use and the association with HIV-related sexual risk. Arch Pediatr Adolesc Med 2007, 161(6):591-596.

22. Corsi KF, Booth RE: HIV sex risk behaviors among heterosexual methamphetamine users: literature review from 2000 to present. Curr Drug Abuse Rev 2008, 1(3):292-296.

23. Wu Z, Sun X, Sullivan SG, Detels R: Public health. HIV testing in China. Science (New York, NY) 2006, 312(5779):1475-1476.

24. China CDC: National behavioral and biological Isurveillance report 2010. Beijing: National Center for AIDS/STD Control and Prevention, China CDC; 2011.

25. Daikos GL, Lai S, Fischl MA: Hepatitis $C$ virus infection in a sexually active inner city population. The potential for heterosexual transmission. Infection 1994, 22(2):72-76.

26. Feldman JG, Minkoff H, Landesman S, Dehovitz J: Heterosexual transmission of hepatitis C, hepatitis B, and HIV-1 in a sample of inner city women. Sex Transm Dis 2000, 27(6):338-342.

27. Tohme RA, Holmberg SD: Is sexual contact a major mode of hepatitis $C$ virus transmission? Hepatology (Baltimore, Md) 2010, 52(4):1497-1505.

doi:10.1186/1471-2458-14-172

Cite this article as: Liao et al.: Syndemics of syphilis, HCV infection, and methamphetamine use along the east coast of China. BMC Public Health 2014 14:172.

\section{Submit your next manuscript to BioMed Central and take full advantage of:}

- Convenient online submission

- Thorough peer review

- No space constraints or color figure charges

- Immediate publication on acceptance

- Inclusion in PubMed, CAS, Scopus and Google Scholar

- Research which is freely available for redistribution 